\title{
PULMONARY MANIFESTATIONS OF EARLY RHEUMATOID ARTHRITIS ON HRCT THORAX
}

\author{
Jayashree Mohanty1, Mamata Singh2, Santosh Kumar Elluru³
}

1 Professor and HOD, Department of Radiodiagnosis, Srirama Chandra Bhanja Medical College and Hospital, Cuttack, Odisha, India. 2Assistant Professor, Department of Radiodiagnosis, Srirama Chandra Bhanja Medical College and Hospital, Cuttack, Odisha, India. 3Junior Resident, Department of Radiodiagnosis, Srirama Chandra Bhanja Medical College and Hospital, Cuttack, Odisha, India.

\section{BACKGROUND}

ABSTRACT

Rheumatoid arthritis (RA) is a common systemic disease that manifests as inflammatory arthritis of multiple joints and produces a wide variety of extra articular manifestations. It affects approximately $1 \%$ of adult population.(1) We wanted to determine the prevalence of airway and parenchymal abnormalities in diagnosed patients of RA with arthritic symptom onset of less than 2 years and to correlate these with clinical measures and laboratory tests.

\section{METHODS}

This is descriptive study of 72 patients with early RA (symptom duration <2 years) diagnosis, 55 females and 17 males, mean age 37.5 years, who underwent high resolution computed tomography (HRCT) thorax. HRCT findings were correlated with clinical and laboratory data.

\section{RESULTS}

Twenty (28\%) patients reported respiratory symptoms: dry cough (17), dyspnoea (11), cough with expectoration (4) and chest pain (4). Rheumatoid Factor (RF) was positive in 51 (71\%) and Anti-CCP Antibody (ACCP) was positive in 54 (75\%). The prevalence of HRCT abnormalities was as follows: ground glass opacities (GGO) 19.4\%, reticular changes $12.5 \%$, honeycombing 6.9\%, bronchiectasis 5.5\%, consolidation 4.2\%. 8 (36\%) patients with HRCT findings did not have any pulmonary symptoms. The following were diagnosed on HRCT: NSIP in 13, UIP in 4, OP in 2, FB in 1 patient. Pulmonary symptoms, smoking, ACCP antibodie s correlated well with RA-ILD.

\section{CONCLUSIONS}

Pulmonary involvement in early RA is common and it involves both pulmonary parenchyma and airways. Significant proportion of patients with pulmonary involvement on HRCT were asymptomatic. GGO and NSIP were the most common HRCT abnormalities. Patients who are smokers, belong to female gender, have pulmonary symptoms \& test positive for ACCP tend to have a higher incidence of ILD. Poor prediction of pulmonary involvement by symptomaticity, RF and ACCP, makes HRCT thorax an important modality for detection of pulmonary involvement in early RA patients. This may allow institution of more aggressive therapy geared towards the prevention of end stage fibrotic lung disease.

\section{KEY WORDS}

Pulmonary Involvement, ILD, Early RA

HOW TO CITE THIS ARTICLE: Mohanty J, Singh M, Elluru SK. Pulmonary manifestations of early rheumatoid arthritis on HRCT thorax. J. Evolution Med. Dent. Sci. 2019;8(26):2097-2100, DOI: 10.14260/jemds/2019/461

\section{BACKGROUND}

Rheumatoid arthritis (RA) is a common systemic disease that manifests as inflammatory arthritis of multiple joints and produces a wide variety of extra articular manifestations. It affects approximately $1 \%$ of adult population(1) worldwide and occurs more commonly in females with female to male ratio of 2-3:1. Some ethnic population like Latin America show even more female predominance in the ratio of 6-8:1.

Due to its systemic nature, RA may result in a variety of extra articular manifestations. Previous studies have shown

'Financial or Other Competing Interest': None.

Submission 01-03-2019, Peer Review 14-06-2019,

Acceptance 21-06-2019, Published 01-07-2019.

Corresponding Author:

Santosh Kumar Elluru,

Department of Radiodiagnosis,

SCBMCH, Cuttack-753007,

Odisha,

India.

E-mail: elluru.santhosh.kumar@gmail.com

DOI: 10.14260/jemds/2019/461 that nearly one third of patients with RA demonstrate some type of extra-articular manifestation involving skin, eye, heart and lung.(2) Extra articular manifestations may occur during the clinical course or even prior to the onset of arthritis. They are the major cause of mortality, infection as the leading cause of death (25\%), followed by cardiac and pulmonary disease (18\%), with renal and gastrointestinal disease lower in frequency $(10 \%)$.

Lung disease is the second most common cause of death in RA.(3) In most of the cases joint symptoms of RA precede the onset of pulmonary symptoms. In few cases however, lung manifestations may precede the onset of joint symptoms, even by several years. This is particularly true for Nonspecific interstitial pneumonia (NSIP), pleuritis, and occasionally obliterative bronchiolitis. RA involves pulmonary parenchyma, airways, pleura and pulmonary vasculature.

Parenchymal involvement in the form of interstitial lung disease (ILD) is one of the important pulmonary manifestations of RA like other connective tissue diseases (CTDs). Nonspecific interstitial pneumonia (NSIP) pattern 
predominates in most CTD-ILD whereas usual interstitial pneumonia (UIP) pattern is more common in RA.

Conditions affecting both the upper and lower airways can occur in patients with rheumatoid arthritis. Upper airway manifestations include rheumatoid nodules on the vocal cords, vasculitis affecting the recurrent laryngeal or vagus nerve leading to vocal cord paralysis, or arthritis of the cricoarytenoid joint. Lower airway disease may include bronchial hyperresponsiveness, bronchiolitis or bronchiectasis.

The hazard ratio for development of clinically evident ILD in RA (Compared to individuals without RA) was 8.96.(4) Moreover, the risk of death for RA patients with ILD was 3 times higher than in RA patients without ILD, and median survival after ILD diagnosis was only 2.6 years.(4) Given the morbidity/mortality associated with progressive/advanced RA-ILD, early diagnosis and aggressive management are critical.

Many patients of RA-ILD do not present with pulmonary symptoms and the restricted activity of these patients due to arthritis makes the pulmonary involvement relatively asymptomatic and hence poses difficulty and delay in diagnosis. As development of pulmonary disease usually postdates the onset of joint symptoms by only $0-5$ years(57), surveillance of patients with recent onset of RA offers the potential for early detection of lung disease. This may increase our understanding of the natural history of ILD and determine which parameters predict the presence and progression of disease. Its detection by imaging (high resolution computed tomography-HRCT), serum markers, pulmonary function tests (PFT) and other modalities is of paramount importance for early detection and management.

There is enough literature on pulmonary involvement in RA patients, but not much study has been done in early RA patients and their HRCT patterns of interstitial disease.

The aim of this study is to know the prevalence and patters of pulmonary involvement in early RA patients by HRCT thorax and to correlate them with clinical and laboratory parameters.

\section{METHODS}

\section{Patient Selection}

Patients presenting to rheumatology clinic and admitted to rheumatology ward of SCB Medical College and Hospital, Cuttack, fulfilling the 2010 ACR/EULAR diagnostic criteria of RA with arthritic symptom onset less than 2years were included in the study. Pregnant patients, patients with other CTDs, other pulmonary diseases like tuberculosis, pneumoconiosis, neoplasm, etc., were excluded from the study. This Descriptive study was conducted after obtaining permission from Ethics Committee of SCB Medical College and Hospital. Informed consent was taken from all subjects before enrolling them into the study.

\section{Clinical and Laboratory Assessment}

Pulmonary symptoms of the subjects were elicited and basic haematological parameters and serum RA markers i.e., RF and ACPA were done.

\section{HRCT Thorax}

Spaced HRCT thorax was done in all subjects in GE Bright Speed 16 slice 3rd generation CT scanner in supine position at end inspiration. Prone scans were done to differentiate basal atelectasis and true posterior lung pathology. Two independent radiologists read the images. Abnormalities on HRCT included reticulations, ground glass opacities, honeycombing, bronchiectasis, consolidation, nodules. According to the predominant findings, each subject was reported as-

1. Normal- No parenchymal or airway abnormalities

2. NSIP- B/L subpleural, basal and lower lobe predominant GGO, reticulations with or without subpleural sparing and traction bronchiectasis.

3. UIP-B/L subpleural, basal and lower lobe predominant honeycombing, reticulations with traction bronchiectasis.

4. OP- B/L patchy arc like/subpleural/peribronchovascular areas of consolidation and/or GGO.

5. Bronchiectasis- Dilated ectatic airways (signet ring sign)/airways reaching till $1 \mathrm{~cm}$ from pleural surface.

6. Rheumatoid nodules- solitary/multiple parenchymal or pleural based nodules, especially if associated with subcutaneous rheumatoid nodules.

\section{Statistical Analysis}

Microsoft word was used to generate tables and data was entered in Microsoft Excel spreadsheet to produce master chart. For descriptive statistics, Statistical Package for Social Sciences was used. Continuous data were described as mean and range, and categorical variables as numbers. To analyse categorical data, chi square test was performed.

\section{RESULTS}

A total of 72 early RA patients with arthritic symptom onset less than 2 years were taken into the study group. Majority of the subjects lie in the age group of 31 to 40 years (52.8\%), followed by age group of 41-50 years (25\%) and least in the age group of above 60 years (1.4\%). Age range of the study was 24 to 64 years with mean age of 37.53 years. Female subjects constituted the bulk with $76.4 \%$ with males accounting for only $23.6 \%$. $20(28 \%)$ had at least one respiratory symptom and 52 (72\%) didn't had any respiratory symptoms. Most common symptom was dry cough followed by dyspnoea. Other symptoms noted included cough with expectoration and chest pain. Only 8 (11\%) patients out of 72 patients had history of tobacco smoking, out of which only 1 was female. RF and ACPA positivity was $71 \%$ and $75 \%$ respectively. Double positive and double negative rates were $23 \%$ and $7 \%$.

\section{Chest Radiograph}

Chest radiograph was abnormal in $10(14 \%)$ subjects. Reticular opacities were the most common finding (Noted in 4 patients), followed by GGO and consolidation (Noted in 2 subjects each). Other findings were nodules (Noted in 1 patient) and features of bronchiectasis such as ring shadows or tram track shadows (Noted in 1 patient).

\section{HRCT Thorax}

25 (35\%) out of 72 subjects had abnormal findings in HRCT Thorax. GGO was noted in 14 (19.4\%) subjects, reticular changes in 9 (12.5\%), honeycombing in 5 (6.9\%), 
consolidation in $3(4.2 \%)$, bronchiectasis in $4(5.5 \%)$, micronodules in 1 (1.4\%), rheumatoid nodules in $1(1.4 \%)$, mosaic perfusion in $5(6.9 \%)$ subjects. All the patients in whom chest X-Ray had findings showed findings in HRCT thorax also. But in 15 patients who had findings in HRCT thorax had no findings in chest X-Ray, i.e., $60 \%$ of patients with pulmonary involvement on HRCT thorax had a normal chest X-ray.

\begin{tabular}{|c|c|c|}
\hline HRCT Abnormalities & Frequency & Percentage \\
\hline Ground glass opacities & 14 & 19.4 \\
\hline Reticular changes & 9 & 12.5 \\
\hline Honeycombing & 5 & 6.9 \\
\hline Consolidation & 3 & 4.2 \\
\hline Bronchiectasis & 4 & 5.5 \\
\hline Micronodules & 1 & 1.4 \\
\hline Rheumatoid nodules & 1 & 1.4 \\
\hline Mosaic perfusion & 5 & 6.9 \\
\hline \multicolumn{2}{|c|}{ Table 1. HRCT Thorax Findings in Early RA Patients } \\
\hline
\end{tabular}

\begin{tabular}{|c|c|c|}
\hline & HRCT + & HRCT - \\
\hline Symptomatic & 16 & 5 \\
\hline Asymptomatic & 8 & 43 \\
\hline Total & 24 & 48 \\
\hline Table 2. Pulmonary Symptoms and HRCT Thorax Correlation \\
(p value=0.000001)
\end{tabular}

\begin{tabular}{|c|c|c|c|c|c|c|}
\hline & Total & Symptomatic & RF + & ACCP+ & Female & Male \\
\hline NSIP & 13 & 8 & 7 & 10 & 11 & 2 \\
\hline UIP & 4 & 3 & 3 & 4 & 3 & 1 \\
\hline OP & 2 & 1 & 2 & 2 & 2 & 0 \\
\hline FB & 1 & 0 & 1 & 0 & 1 & 0 \\
\hline BX & 4 & 3 & 3 & 2 & 2 & 2 \\
\hline RN & 1 & 1 & 0 & 1 & 1 & 0 \\
\hline Total & 25 & 16 & 16 & 19 & 20 & 5 \\
\hline \multicolumn{7}{|c|}{ Table 3. HRCT Thorax Diagnosis Vs Other Clinical and } \\
Laboratory Parameters \\
\hline
\end{tabular}

\begin{tabular}{|c|c|c|c|}
\hline & ILD + & ILD - & p Value \\
\hline Females & 16 & 39 & \multirow{2}{*}{0.3494} \\
\hline Males & 3 & 14 & \\
\hline Smoking + & 4 & 4 & \multirow{2}{*}{0.1080} \\
\hline Smoking - & 15 & 49 & \\
\hline $\mathrm{RF}+$ & 12 & 39 & \multirow{2}{*}{0.3909} \\
\hline RF - & 7 & 14 & \\
\hline ACPA + & 16 & 38 & \multirow{2}{*}{0.2798} \\
\hline ACPA - & 3 & 15 & \\
\hline
\end{tabular}

Table 4. ILD Correlation with Clinical and Laboratory Parameters

\begin{tabular}{|c|c|c|c|c|c|c|}
\hline \multirow{2}{*}{ References } & \multirow{2}{*}{ Year } & $\begin{array}{c}\text { No. of } \\
\text { Patients }\end{array}$ & \multicolumn{5}{|c|}{ UIP } & NSIP & OP & Others \\
\hline Tanaka et al.(5) & 2004 & 63 & $\begin{array}{c}26 \\
(50 \%)\end{array}$ & $\begin{array}{c}19 \\
(36 \%)\end{array}$ & $\begin{array}{c}5 \\
(10 \%)\end{array}$ & $\begin{array}{c}2 \\
(4 \%)\end{array}$ \\
\hline Kim et al.(6) & 2012 & 84 & $\begin{array}{c}20 \\
(24 \%)\end{array}$ & $\begin{array}{c}19 \\
(23 \%)\end{array}$ & $\begin{array}{c}1 \\
(1 \%)\end{array}$ & $\begin{array}{c}43 \\
(52 \%)\end{array}$ \\
\hline Hozumi et al.(7) & 2013 & 51 & $\begin{array}{c}14 \\
(27 \%)\end{array}$ & $\begin{array}{c}37 \\
(73 \%)\end{array}$ & & \\
\hline Kelly et al.(8) & 2014 & 230 & $65 \%$ & $24 \%$ & $5 \%$ & $6 \%$ \\
\hline Our Study & 2018 & 72 & $\begin{array}{c}4 \\
(17 \%)\end{array}$ & $\begin{array}{c}13 \\
(54 \%)\end{array}$ & $\begin{array}{c}2 \\
(8 \%)\end{array}$ & $\begin{array}{c}6 \\
(25 \%)\end{array}$ \\
\hline \multicolumn{7}{|c|}{ Table 5. HRCT Disease Patterns in Different Studies on RA } \\
Patients. ${ }^{(9)}$
\end{tabular}

Out of the 25 patients in whom HRCT was abnormal, $16(64 \%)$ had one or more pulmonary symptoms and remaining $8(36 \%)$ subjects didn't complain of any pulmonary symptoms. Thus, a significant proportion of patients without any pulmonary symptoms had HRCT abnormalities. Also 5 patients who had pulmonary symptom/s had a normal HRCT thorax.

\section{ILD Patterns}

Depending on the HRCT abnormalities and patterns, each case was classified and was designated to a particular ILD pattern. 13 cases $(18.1 \%)$ were diagnosed as NSIP, 4 cases $(5.5 \%)$ as UIP, 2 cases $(2.8 \%)$ as organizing pneumonia Incidence of ILD in male and female patients was 17.6 and $29.1 \%$ respectively. $61.54 \%$ (8 out of 13 ) of NSIP patients, $75 \%$ ( 3 out of 4 ) of UIP patients, $50 \%$ (1 out of 2 ) of OP patients had at least one pulmonary symptom. ILD incidence was $50 \%$ in smokers versus $23.4 \%$ in non-smokers. $53.84 \%$ (7 out of 13) of NSIP patients, 75\% (3 out of 4 ) of UIP patients, $100 \%$ (2 out of 2 ) patients of OP had their serum RF positive. $63.15 \%$ (12 out of 19) of all ILD patients had RF positivity. While $73.58 \%$ (39 out of 53 ) of early RA patients without ILD had RF positivity. 76.92\% (10 out of 13) of NSIP patients, $100 \%$ ( 4 out of 4 ) of UIP patients, $100 \%$ (2 out of 2 ) patients of OP had their serum anti CCP Ab positive. Overall 84.21\% (16 out of 19) of all ILD patients had anti CCP Ab positivity. While $71.70 \%$ (38 out of 53) of early RA patients without ILD had anti CCP Ab positivity.

\section{DISCUSSION}

RA being a systemic disease, extra articular manifestations are common. Pulmonary involvement is common even in early RA patients as demonstrated by our study.

Many studies are available in the literature regarding pulmonary involvement in long standing RA, but only a handful of studies are there in the literature on early RA. To understand the natural course and complications related to lung in RA, we need more studies which focus on the early RA patients. Also, the incidence, natural course and complications of RA is partly dependent on the region, ethnicity, life style of the patients. There are no studies done on East Indian population regarding the same. Therefore, we decided to do this study in the East Indian population and included only early RA patients with arthritic symptoms onset less than 2 years.

Our study has described all the HRCT abnormalities including both parenchymal and airway abnormalities in early RA patients with arthritic symptom onset less than 2 years and all ILD cases have been designated different HRCT patterns of ILD.

Pulmonary involvement was noted in almost one third (35\%) of the patients, out of which a significant proportion (36\%) of patients didn't had any pulmonary complaints. Females had higher incidence of pulmonary involvement than males. Most common HRCT abnormality was GGO. Chest radiograph was not sensitive in detecting pulmonary involvement.

In Gabbay et al(10) study, 33\% of patients showed evidence of ILD on HRCT with GGO in $28 \%$, reticulations in $39 \%$, honeycombing in $6 \%$, bronchiectasis in $6 \%$, traction bronchiectasis in $6 \%$, consolidation in $3 \%$. Wilsher et al(11) reported an incidence of GGO of $23 \%$, reticulations of $18 \%$, bronchiectasis of $48 \%$ and consolidation of $7 \%$ in HRCT. In a study conducted by Mori et al,(12) GGO was noted in $27 \%$, reticulations in $11.9 \%$, honeycombing in $8.7 \%$, consolidation in $4 \%$ and nodules in $10.3 \%$ in HRCT on subjects of both early and longstanding RA patients. In Liote et al(13) study CT evidence of pulmonary disease was present in 50\% of RA patients, but only $10 \%$ of these patients had clinical symptoms. 
In a review of literature by Suda, UIP is the most common HRCT pattern, ranging from 24 to $65 \%$, followed by NSIP (Ranging $23 \%$ to $73 \%$ ) and OP (Ranging $1 \%$ to $10 \%$ ). ${ }^{(9)}$

In our study ILD was noted in $26.4 \%$ of patients, NSIP was the most common ILD. On contrary UIP is the most common ILD in RA patients according to literature. This difference might be due to inclusion of only early RA patients in our study or due to ethnic difference in the incidence of ILD in RA. Smokers had 50\% incidence of ILD compared to $23 \%$ in nonsmokers. RF positivity was higher in non-ILD group compared to ILD group, while ACPA positivity was higher in ILD group compared to non-ILD group. However no statistically significant correlation was noted between serum markers and ILD.

Limitations of the study were small sample size; subjects were only from a single institution and non-inclusion of PFT and BAL.

To summarize, pulmonary involvement in early RA is common and it involves both pulmonary parenchyma and airways. Significant proportion of patients with pulmonary involvement on HRCT were asymptomatic. NSIP was the most common ILD in contrast to UIP in long standing cases. Though there was positive correlation of female gender, smoking and ACPA with pulmonary involvement, it was statistically not significant.

\section{CONCLUSIONS}

Pulmonary involvement occurs early in patients of RA. Absence of pulmonary symptoms in a significant proportion of patients combined with poor prediction of pulmonary involvement by RF and ACCP, and poor sensitivity of chest Xray makes HRCT thorax an important modality for detection of pulmonary involvement in early RA patients. This may allow institution of more aggressive therapy geared towards the prevention of end stage fibrotic lung disease.

\section{REFERENCES}

[1] Gabriel SE. The epidemiology of rheumatoid arthritis. Rheum Dis Clin North Am 2001;27(2):269-81.

[2] Bharadwaj A, Haroon N. Interstitial lung disease and neuropathy as predominant extra - articular manifestations in patients with rheumatoid arthritis: a prospective study. Med Sci Monit 2005;11(10):CR498-CR502.
[3] Suzuki A, Ohosone Y, Obana M, et al. Cause of death in 81 autopsied patients with rheumatoid arthritis. J Rheumatol 1994;21(1):33-6.

[4] Bongartz T, Nannini C, Medina-Velasquez YF, et al. Incidence and mortality of interstitial lung disease in rheumatoid arthritis: a population-based study. Arthritis and Rheumatism 2010;62(6):1583-91.

[5] Tanaka N, Kim JS, Newell JD, et al. Rheumatoid arthritis-related lung diseases: CT findings. Radiology 2004;232(1):81-91.

[6] Kim EJ, Elicker BM, Maldonado F, et al. Usual interstitial pneumonia in rheumatoid arthritisassociated interstitial lung disease. Eur Respir J 2010;35(6):1322-8.

[7] Hozumi H, Nakamura $\mathrm{Y}$, Johkoh T, et al. Acute exacerbation in rheumatoid arthritis-associated interstitial lung disease: a retrospective case control study. BMJ Open 2013;3(9):e003132.

[8] Kelly CA, Saravanan V, Nisar M, et al. Rheumatoid arthritis-related interstitial lung disease: associations, prognostic factors and physiological and radiological characteristics - a large multicentre UK study. Rheumatology (Oxford) 2014;53(9):1676-82.

[9] Suda T. Up-to-Date information on rheumatoid arthritis-associated interstitial lung disease. Clin Med Insights Circ Respir Pulm Med 2016;9(Suppl 1):15562.

[10] Gabbay E, Tarala R, Will R, et al. Interstitial lung disease in recent onset rheumatoid arthritis. Am J Respir Crit Care Med 1997; 156(2 Pt 1):528-35.

[11] Wilsher M, Voight L, Milne D, et al. Prevalence of airway and parenchymal abnormalities in newly diagnosed rheumatoid arthritis. Respir Med 2012;106(10):1441-6.

[12] Mori S, Koga Y, Sugimoto M. Small airway obstruction in patients with rheumatoid arthritis. Mod Rheumatol 2011;21(2):164-73.

[13] Liote H. Pulmonary manifestation of rheumatoid arthritis. Revue Des Maladies Respiratoires 2008;25(8):973-88. 